\title{
Reciprocal regulation of TEAD4 and CCN2 for the trophectoderm development of the bovine blastocyst
}

\author{
Hiroki Akizawa ${ }^{1,2}$, Ken Kobayashi ${ }^{3}$, Hanako Bai ${ }^{1}$, Masashi Takahashi ${ }^{1}$, Shinjiro Kagawa ${ }^{1}$, \\ Hiroaki Nagatomo ${ }^{1,+}$ and Manabu Kawahara ${ }^{1}$ \\ ${ }^{1}$ Laboratory of Animal Genetics and Reproduction, Research Faculty of Agriculture, Hokkaido University, Sapporo, \\ Japan, ${ }^{2} J a p a n$ Society for the Promotion of Science (JSPS Research Fellow), Tokyo, Japan and ${ }^{3}$ Laboratory of Cell \\ and Tissue Biology, Research Faculty of Agriculture, Hokkaido University, Sapporo, Japan \\ Correspondence should be addressed to M Kawahara; Email: k-hara@anim.agr.hokudai.ac.jp
}

${ }^{\dagger}$ (Hiroaki Nagatomo is now at Department of Biotechnology, Faculty of Life and Environmental Science, University of Yamanashi, Kofu, Japan)

\begin{abstract}
The first segregation at the blastocyst stage is the symmetry-breaking event to characterize two cell components; namely, inner cell mass (ICM) and trophectoderm (TE). TEA domain transcription factor 4 (TEAD4) is a well-known regulator to determine TE properties of blastomeres in rodent models. However, the roles of bovine TEAD4 in blastocyst development have been unclear. We here aimed to clarify the mechanisms underlining TE characterization by TEAD4 in bovine blastocysts. We first found that the TEAD4 mRNA expression level was greater in TE than in ICM, which was further supported by TEAD4 immunofluorescent staining. Subsequently, we examined the expression patterns of TE-expressed genes; CDX2, GATA2 and CCN2, in the TEAD4-knockdown (KD) blastocysts. These expression levels significantly decreased in the TEAD4 KD blastocysts compared with controls. Of these downregulated genes, the CCN2 expression level decreased the most. We further analyzed the expression levels of TE-expressed genes; CDX2, GATA2 and TEAD4 in the CCN2 KD blastocysts. Strikingly, the CCN2 KD blastocysts showed the downregulation of CDX2, GATA2 and TEAD4. Furthermore, the ratio of TE-to-ICM cell numbers in the CCN2 KD blastocysts significantly decreased compared to controls. To our knowledge, this is the first study showing the regulation of CCN2 expression thorough TEAD4 in mammalian embryos. Not only that, this study also provides evidence that reciprocal regulation of TEAD4 and CCN2 is required for TE development with appropriate gene expression in bovine blastocysts.

Reproduction (2018) 155 563-571
\end{abstract}

\section{Introduction}

The blastocyst stage is a crucial period for mammalian preimplantation development because the first two lineages, namely the inner cell mass (ICM) and trophectoderm (TE), are distinctly established by this stage (Arnold \& Robertson 2009). These cell lineages are fundamental for proper embryo and extraembryonic tissue formation (Arnold \& Robertson 2009). As TE cells are derived from pluripotent undifferentiated blastomeres under the regulation of signal transduction pathways controlled strictly, the perturbation of these pathways could cause early embryonic lethal phenotype in mice (Chawiengsaksophak et al. 1997, Nichols et al. 1998, Mitsui et al. 2003).

TE is a monolayer of epithelial-like cell populations surrounding the outer part of the blastocyst, whose destination is determined by the expression level of a transcription factor, caudal type homeobox 2 (CDX2) (Strumpf et al. 2005). Hence, CDX2 is recognized as the most important factor in acquiring TE properties for the blastocyst development. As a potent evidence of this, ectopic expression of CDX2 in embryonic stem cells allows them to convert to trophoblast stem (TS) like cells (Niwa et al. 2005). One of the upstream regulators of CDX2 in mouse embryos is TEA domain transcription factor 4 (TEAD4) (Yagi et al. 2007). TEAD4 contributes to the establishment of a robust transcriptional network in TE by regulating not only CDX2 but also many other genes critical for normal development, such as Eomesodermin (Eomes) (Yagi et al. 2007), heart and neural crest derivatives expressed 1 (Hand1) (Nishioka et al. 2009) and GATA-binding protein 3 (Gata3) (Ralston et al. 2010). Thus, TEAD4 is an essential mediator for the proper TE differentiation, whose loss leads to failure of TS cell line establishment in mice (Yagi et al. 2007). Although the important genes such as CDX2 and TEAD4 are commonly expressed at the blastocyst stage in most mammals (Berg et al. 2011, Home et al. 2012, Niakan \& Eggan 2013, Bou et al. 2017), the regulatory mechanisms 
appear to vary among species (Berg et al. 2011, Kuijk et al. 2012). For example, bovine TEAD4 shows unique function to activate the ruminant-specific pregnancy recognition factor, interferon tau (Kusama et al. 2016). Therefore, to precisely evaluate the roles of TEAD4 in the first cell segregation at the blastocyst stage, the analyses dedicated to each species are required.

$\mathrm{CCN}$ family member 2 (CCN2) is well-known as one of potent downstream factors of TEAD4 in mammalian somatic cells, and this regulation of $\mathrm{CCN} 2$ expression by TEAD4 controls cell proliferation (Zhao et al. 2008). Further, we previously found CCN2 mRNA was more predominantly expressed in TE cells than in ICM cells in the bovine blastocyst (Nagatomo et al. 2013). This sitepredominant CCN2 expression might be associated with the TE development through TEAD4 in cattle. However, this interaction between TEAD4 and CCN2 has not been proved in mammalian preimplantation embryos including cattle.

To elucidate the relationship between TEAD4 and CCN2 in the bovine blastocyst, we conducted the shRNA-mediated RNA interference for the suppression of TEAD4 transcripts. Knockdown (KD) of TEAD4 induced significant downregulation of TE-expressed genes, CDX2, GATA2 and CCN2. Among these downregulated genes, the CCN2 expression level was most altered in the TEAD4 KD blastocysts. Next, we performed CCN2 $\mathrm{KD}$ in bovine embryos, in which the expression levels of TE-expressed genes significantly decreased as well as in the TEAD4 KD blastocysts. Notably, the TEAD4 expression level in the CCN2 KD blastocysts significantly decreased. The CCN2 KD also decreased the ratio of TE to ICM cell numbers compared to the control blastocysts. Our results provide the first demonstration that the CCN2 expression could be influenced by TEAD4 in mammalian embryos. Not only that, reciprocal regulation of TEAD4 and CCN2 was required for stable expression of TE differentiation regulators, CDX2 and GATA2 in bovine blastocyst. Taken together, our findings offer the novel insights into the molecular circuitry governing the TE lineage differentiation through TEAD4 in bovine blastocyst development.

\section{Materials and methods}

All experimental protocols were approved by the Regulatory Committee for the Care and Use of Laboratory Animals, Hokkaido University.

\section{Preparation of bovine embryos by in vitro fertilization}

Bovine oocyte retrieval, in vitro oocyte maturation, fertilization and subsequent in vitro embryo culture were performed as described previously (Nagatomo et al. 2013). Briefly, cumulus-oocyte complexes (COCs) collected from slaughterhouse-derived ovaries were matured by culturing in TCM-199 medium (Thermo Fisher Scientific) at $38.5^{\circ} \mathrm{C}$ in a humidified atmosphere of $5 \% \mathrm{CO}_{2}$ in air for $20-22 \mathrm{~h}$. In vitromatured oocytes were transferred to Brackett and Oliphant (BO) medium (Brackett \& Oliphant 1975) containing $2.5 \mathrm{mM}$ theophylline (Wako Pure Chemical Industries) and $7.5 \mu \mathrm{g} /$ $\mathrm{mL}$ heparin sodium salt (Nacalai Tesque, Inc., Kyoto, Japan). Subsequently, frozen-thawed semen was centrifuged at $600 \mathrm{~g}$ for $7 \mathrm{~min}$ in $\mathrm{BO}$ medium, and the spermatozoa were added to the COCs at a final concentration of $5 \times 10^{6} \mathrm{cell} / \mathrm{s} / \mathrm{mL}$. After $12 \mathrm{~h}$ of incubation, the presumptive zygotes were denuded by pipetting and cultured in mSOFai medium (Aono et al. 2013) at $38.5^{\circ} \mathrm{C}$ in a humidified atmosphere of $5 \% \mathrm{CO}_{2}$ and $5 \% \mathrm{O}_{2}$ in air for 8 days.

\section{Quantitative reverse-transcription PCR}

Total RNA from five oocytes or embryos per biological replicate was isolated using the ReliaPrep RNA Cell Miniprep System (Promega), following the manufacturer's instructions. To obtain purely isolated ICM and TE for RNA preparation, blastocyst separation procedures were carried out according to our previous study (Nagatomo et al. 2013). Briefly, to evaluate the TEAD4 mRNA expression level of only ICM, chemical isolation with detergent solution containing $0.2 \%$ (v/v) Triton X-100 in PBS was performed. This isolated ICM cell sample contained none of the TE cells as confirmed previously (Nagatomo et al. 2013). We separated TE from blastocysts using a micromanipulator equipped with a microsurgical blade (Feather, Osaka, Japan) under an inverted microscope (Olympus). The cDNA syntheses from ICM, TE, and whole embryo were conducted using ReverTra Ace qPCR RT Master Mix (Toyobo, Osaka, Japan). Quantitative PCR (qPCR) was performed after preparing the reaction mixtures in THUNDERBIRD SYBR qPCR Mix (Toyobo). The primer sets used for qPCR analysis were listed in Supplementary Table 1 (see section on supplementary data given at the end of this article). Thermal cycling conditions consisted of 1 cycle at $95^{\circ} \mathrm{C}$ for $30 \mathrm{~s}$ (denaturation), followed by 50 cycles at $95^{\circ} \mathrm{C}$ for $10 \mathrm{~s}$ (denaturation), annealing temperature corresponding to each primer set for $15 \mathrm{~s}$ (primer annealing) and $72^{\circ} \mathrm{C}$ for $30 \mathrm{~s}$ (extension). The TE-expressed genes examined in the present study were selected from the genes that played important roles for TE development in mouse blastocysts (Gulnaar et al. 1998, Niwa et al. 2005, Yagi et al. 2007, Ralston et al. 2010, Home et al. 2017) and were examined using TE of bovine blastocysts in our previous study (Nagatomo et al. 2013). Relative mRNA abundance was calculated by the $\triangle \triangle \mathrm{Ct}$ method, with $H 2 A F Z$ ( $\mathrm{H} 2 \mathrm{~A}$ histone family member $\mathrm{Z}$ ) as the reference gene in each sample. The experiments were replicated three times.

\section{Immunofluorescence and confocal microscopy}

Primary antibodies included rabbit anti-CCN2 antibody (1:300, ab6992; Abcam), rabbit anti-CDX2 antibody (1:200, ab76541; Abcam) and mouse anti-TEAD4 antibody $(1: 1500$, ab58310; Abcam). Secondary antibodies were Alexa Fluor 555 goat anti-rabbit IgG polyclonal (ab150082; Thermo Fisher Scientific, Inc.) and Alexa Fluor 488 goat antimouse IgG H\&L (ab150113, Thermo Fisher Scientific, Inc.). 
The zona pellucida of the oocytes and embryos were removed with $0.05 \%(\mathrm{w} / \mathrm{v})$ Pronase (Sigma-Aldrich). Oocytes and embryos were fixed with $4 \%(\mathrm{w} / \mathrm{v})$ paraformaldehyde (PFA; Wako Pure Chemical Industries) in PBS for $60 \mathrm{~min}$, and then permeabilized for $60 \mathrm{~min}$ with $0.2 \%$ (v/v) Triton $\mathrm{X}-100$ in PBS. Next, the oocytes and embryos were blocked for $45 \mathrm{~min}$ with Blocking One (1:5; Nacalai Tesque, Inc.) diluted in $0.05 \%$ (v/v) Tween 20 in PBS (blocking buffer). Temperature and incubation time during the primary antibody reaction varied depending on the target protein; namely, overnight at $4^{\circ} \mathrm{C}$ for $\mathrm{CCN} 2$, overnight at $37^{\circ} \mathrm{C}$ for CDX2 and $2 \mathrm{~h}$ at $20-22^{\circ} \mathrm{C}$ for TEAD4. After washes in $0.1 \%$ $(\mathrm{v} / \mathrm{v})$ Triton $\mathrm{X}-100$ and $0.3 \%(\mathrm{w} / \mathrm{v})$ bovine serum albumin (Sigma-Aldrich) in PBS for 10 min five times, the oocytes and embryos were incubated for $30 \mathrm{~min}$ at room temperature with each secondary antibody diluted to 1:400 in blocking buffer. Nuclei were counterstained with 25 mg/mL Hoechst 33342 (Sigma-Aldrich) prepared in $0.2 \%(\mathrm{w} / \mathrm{v})$ polyvinyl alcohol in PBS. Fluorescence signals were visualized using a TCS SP5II confocal laser-scanning microscope (Leica).

\section{Whole-mount in situ hybridization (WISH)}

WISH for TEAD4 mRNA in bovine blastocysts was performed as described previously (Nagatomo et al. 2013). Briefly, bovine cDNA fragments encoding TEAD4 transcripts were obtained by PCR amplifications and were transcribed in vitro with digoxigenin (DIG)-labeling using the T7 RNA polymerase for sense and antisense probes (DIG RNA Labeling Mix, Roche Diagnostics). The primer set to prepare sense and antisense probes was identical to that used for qPCR of TEAD4. The expanded blastocysts at day 8 were fixed and stored in $4 \%$ $(\mathrm{w} / \mathrm{v})$ PFA (Wako Pure Chemical Industries) in PBS with $0.1 \%(\mathrm{v} / \mathrm{v})$ Tween 20 (PBT) at $4{ }^{\circ} \mathrm{C}$ for $30 \mathrm{~min}$ to overnight. Fixed embryos were treated with proteinase $\mathrm{K}$ (SigmaAldrich) and placed in 4\% (w/v) PFA, 0.2\% (v/v) EM-grade glutaraldehyde (Sigma-Aldrich) in PBT at room temperature for $20 \mathrm{~min}$. Subsequently, the blastocysts were placed in hybridization buffer (4x saline-sodium citrate (SSC) $(\mathrm{pH}$ 7.0), 50\% (v/v) deionized formamide (Wako Pure Chemical Industries, Ltd.), $100 \mu \mathrm{g} / \mathrm{mL}$ heparin (Wako Pure Chemical Industries, Ltd.), $100 \mu \mathrm{g} / \mathrm{mL}$ salmon sperm DNA (Wako Pure Chemical Industries, Ltd.), $2 \times$ Denhardt's solution and $0.1 \%$ (v/v) Tween 20 (Wako Pure Chemical Industries, Ltd.)) at $60-70^{\circ} \mathrm{C}$ for $3-8 \mathrm{~h}$. After these pretreatments, the blastocysts were subjected to WISH with DIG-labeled riboprobes in hybridization buffer overnight at the same temperature as prehybridization, followed by washing with buffer $(50 \%$ (v/v) formamide, $2 \times$ SSC, $0.1 \%(v / v)$ Tween 20) 3 times at the hybridization temperature. According to the manufacturer's recommended procedure to detect DIG-labeled probes, samples were incubated in NBT/BCIP (nitro blue tetrazolium/ 5-bromo-4-chloro-3-indolyl-phosphate) solution (Roche) until coloration was obtained after incubation with anti-DIG antibodies (Roche). The hybridized embryos were observed in PBS containing $1 \mathrm{mM}$ EDTA and $20 \%$ (v/v) glycerol for bright-field photographing under an inverted microscope (AMEX-1200, AMG, Bothell, WA, USA).

\section{Microinjection of TEAD4 and CCN2 shRNA expression vectors}

Construct preparation for RNA interference and subsequent microinjection into presumptive zygotes were performed as described previously (Akizawa et al. 2016). A shRNA containing antisense/sense regions, an 19-bp loop (5'-CTGTGAAGCCACAGATGGG) and a 6-bp terminator element (5'-TTTTTT) was designed to target nucleotides 913-932 of TEAD4 mRNA (NCBI Reference Sequence XM_010827947) as well as target nucleotides 665-683 of CCN2 mRNA (NCBI Reference Sequence NM_174030). The dsDNA was ligated downstream of the U6 promoter in the pBAsi/mU6 Neo vector (Stratagene, CA, USA). pBAsi/mU6 Neo plasmids targeting nucleotides 5957-5977 of enhanced green fluorescent protein (egfp) sequence (NCBI Reference Sequence NC_025025.1) (shegfp) and plasmids lacking shRNA insert (empty vector) as used in the early studies from other laboratories (Orimo et al. 2005, Andey et al. 2014, Nelson et al. 2014, Li et al. 2015) and our previous reports (Nagatomo et al. 2015, 2016, Akizawa et al. 2016). Twelve hours after insemination, the synthesized shRNA expression constructs targeting TEAD4, CCN2 and egfp, and empty vector (diluted to a final concentration of $10 \mathrm{ng} / \mathrm{mL}$ with $\mathrm{mSOFai}$ medium) were injected into the denuded presumptive zygotes using a Femtolet injection device (Eppendorf, Hamburg, Germany). These presumptive zygotes were cultured to examine the effect of KD on embryonic development until the blastocyst stage.

\section{Statistical analysis}

All experimental data were presented as mean \pm standard error of mean (S.E.M.). In the data on the TEAD4 expression levels among whole embryos, ICM and TE, and the TE-expressed gene expression levels among uninjected, empty and shegfp embryos, significant differences were compared using repeated-measures ANOVA followed by the Tukey's post hoc tests. Other than these experiments, data were statistically analyzed using unpaired Student's $t$ test. Stat View (Abacus Concepts, Inc., Piscataway, NJ, USA) was used for analysis. $P<0.05$ was considered statistically significant.

\section{Results \\ Site specificity of TEAD4 expression in bovine blastocysts}

We first determined the site-dominant TEAD4 expression by WISH and qPCR in bovine blastocysts. Although TEAD4 mRNA was expressed both in TE and ICM (Fig. 1A), the expression level of TEAD4 mRNA in TE was significantly higher than that in ICM (Fig. 1B). Moreover, whole-mount immunofluorescent staining revealed that TEAD4 proteins were predominantly expressed in nuclei of TE cells compared with nuclei of ICM cells (Fig. 1C). This expression pattern was consistent with previous report in cattle (Home et al. 2012), but was different from murine blastocysts (Nishioka et al. 2008). 
A

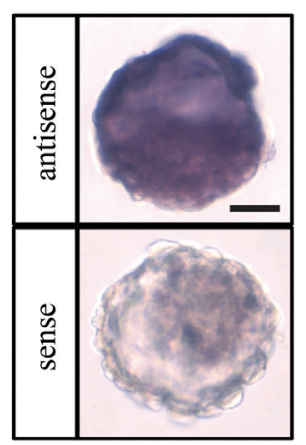

B

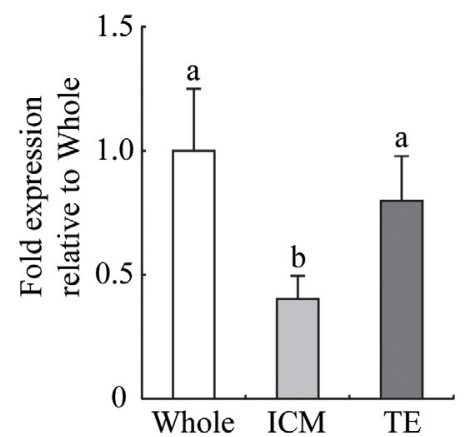

C

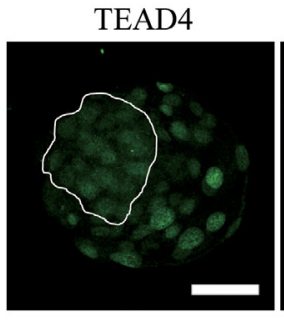

DNA

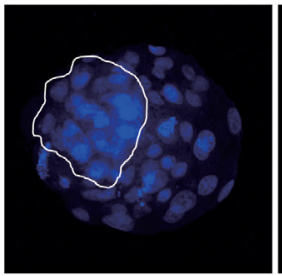

Merge

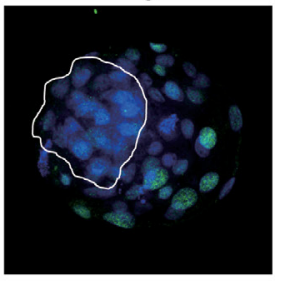

Figure 1 Site-dominant TEAD4 expression status at the mRNA and protein levels in bovine blastocysts. (A) Whole-mount in situ hybridization for TEAD4 mRNA. The results from DIG-labeled antisense and sense riboprobes were represented in the upper and lower panels, respectively. (B) The TEAD4 mRNA expression level in whole embryo, chemically isolated ICM and mechanically isolated mural TE cell samples as described in the method section. The values represent relative expression levels to whole embryo. The results are shown as means \pm S.E.M. (error bars) of three replicate experiments. (C) Immunofluorescent analysis of TEAD4 protein expression in the bovine blastocyst. The experiment was conducted three times, and at least five embryos were analyzed for each experiment. The white lines indicate ICM. (a and b) Different letters indicate significant differences between groups $(P<0.05)$. Scale bar $=100 \mu \mathrm{m}$.

\section{Effects of TEAD4 KD on TE-expressed gene expression in bovine blastocysts}

To uncover the regulation of TE-expressed gene transcription by TEAD4 in bovine embryos, we attempted to suppress TEAD4 mRNA by shRNA-mediated interference. Before the TEAD4 KD experiment, we confirmed that injection of empty or shegfp vectors was non-effective for the expression levels of any genes examined in the subsequent experiments (Fig. 2). There were no significantly differences in expression levels of all the examined genes among uninjected, empty and shegfp embryos, indicating that microinjection procedure and shRNA production have no impact on gene expression status in bovine embryos. Therefore, we regarded empty vector-injected embryos as controls of KD embryos in the subsequent experiments.

The efficiency of TEAD4 KD was validated both at the mRNA and protein levels by $\mathrm{qPCR}$ and immunofluorescent staining respectively (Fig. $3 \mathrm{~A}$ and B). The TEAD4 expression level in the TEAD4 KD blastocysts significantly decreased compared with empty vector-injected controls (Fig. 3A). Moreover, the immunofluorescent signals showing the TEAD4 proteins in the TEAD4 KD embryos were obviously reduced (Fig. 3B). Moreover, to confirm the specificity of shTEAD4 vector, the expression level of TEAD3 that is a member of TEAD family was investigated in the TEAD4 KD embryos (Supplementary Fig. 1). As expected, the TEAD3 in the TEAD4 KD embryos was expressed at comparable level to that in controls (Supplementary Fig. 1). After these validations of $K D$ efficiency, the in vitro development of TEAD4 KD embryos was investigated until the blastocyst stage. There were no significant differences in the rates of cleaved embryos and blastocyst formation between TEAD4 KD embryos and empty vector-injected controls (Table 1), which was consistent with previous report (Sakurai et al. 2017). The qPCR analyses revealed that the TEAD4 KD blastocysts showed significantly suppressed expression levels of 3 TE-expressed genes; CDX2, GATA2 and CCN2 $(P<0.05)$ (Fig. 3C). Among the downregulated genes in the TEAD4 KD blastocysts, CCN2 showed 11-fold decreased expression compared with that of empty vector-injected controls. In the

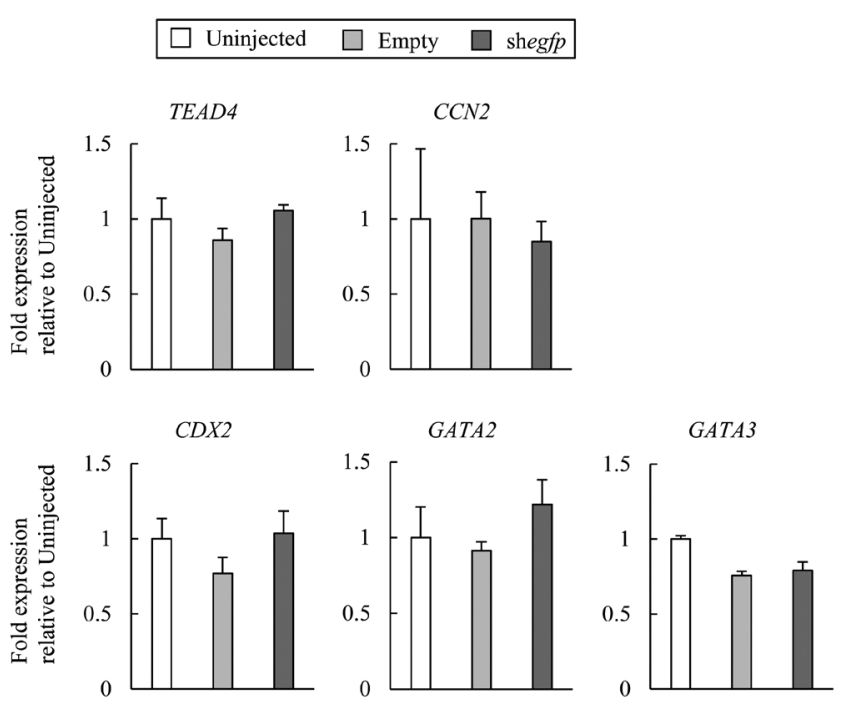

Figure 2 Validation of embryos injected with empty vectors as control in the shRNA-mediated RNA interference system. The mRNA expression levels of TEAD4, CCN2, CDX2, GATA2 and GATA3 were investigated among the following three types of embryos at the blastocyst stage: the uninjected embryos (Uninjected); the embryos that empty vectors were injected into (Empty) and the embryos that vectors targeting egfp sequence were injected into (shegfp). The values represent relative expression levels to Uninjected. The results are shown as means \pm S.E.M. (error bars) of four to five replicate experiments. 
$\mathbf{A}$

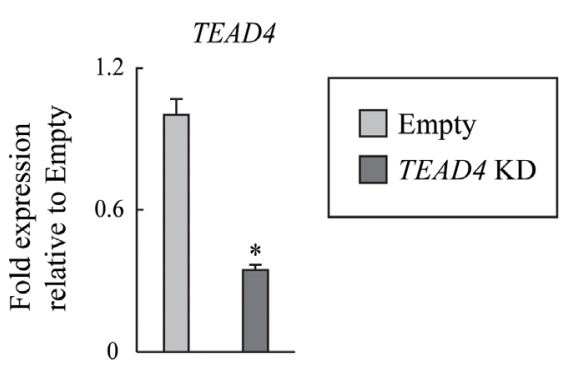

C

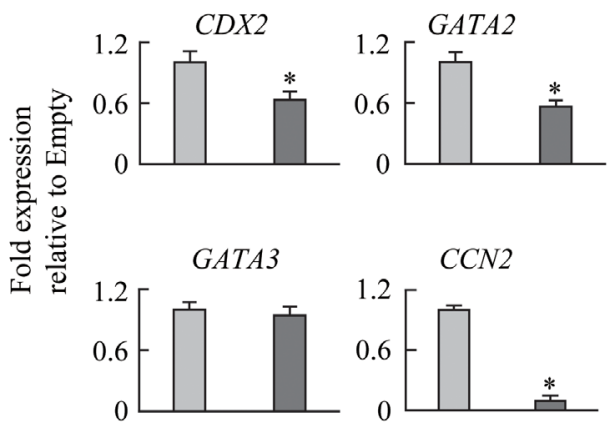

B

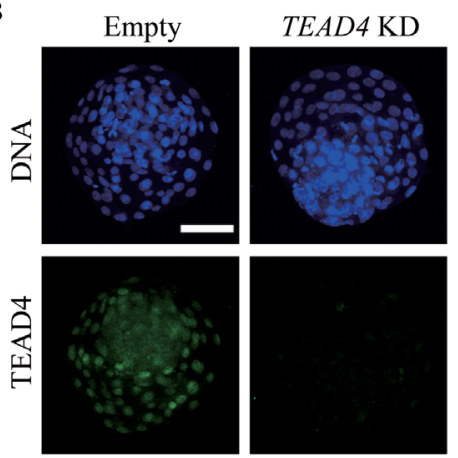

D

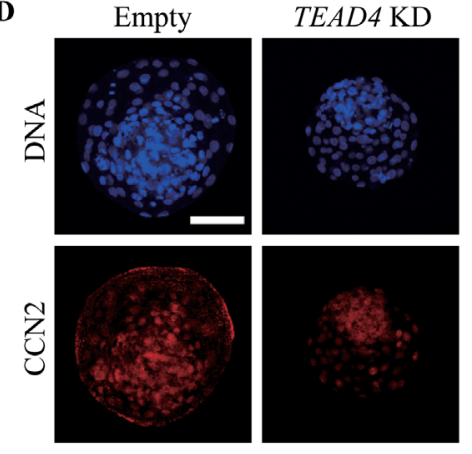

Figure 3 Effects of TEAD4 knockdown (KD) on TE-expressed gene expression in bovine blastocysts. TEAD4 KD efficiency was confirmed in blastocysts both at the mRNA (A) and protein (B) expression levels $(34.5 \%$ compared to Empty). (C) The relative expression levels of four TE-expressed genes in empty vector-injected controls and TEAD4 KD blastocysts (CDX2: 60.0\%, GATA2: $56.2 \%$, GATA3: $94.4 \%$, and CCN2: $9.2 \%$ compared to Empty, respectively). (D) Immunofluorescent analysis of $\mathrm{CCN} 2$ protein expression in the TEAD4 KD blastocyst. Ten embryos were analyzed for each experiment. The values represent relative expression levels to empty vector-injected controls. The results are shown as means \pm S.E.M. (error bars) of three replicate experiments, $* P<0.05$. Scale bar $=100 \mu \mathrm{m}$. protein level, CCN2 expression is suppressed in TE, but not in ICM (Fig. 3D).

\section{Reciprocal regulation of TEAD4 and CCN2 in bovine blastocysts}

Although there are several studies to demonstrate the essential roles of $C C N 2$ expression regulated by TEAD4 in NIH-3T3, MCF10A (Zhao et al. 2008) and human mammary epithelial cells (Lai et al. 2011), the significance of CCN2 expression during preimplantation development has remained unclear. Therefore, we next attempted to suppress CCN2 mRNA by shRNA-mediated interference and validated the CCN2 KD efficiency by assessing CCN2 mRNA and protein expression. The CCN2 expression level significantly decreased in the CCN2 KD blastocysts (Fig. 4A), which was consistent with the reduction of fluorescent signals representing CCN2 proteins (Fig. 4B). Similar to TEAD4 KD, CCN2 KD had no effects on in vitro developmental rates of cleavage and the blastocyst formation (Table 2). Furthermore, we determined the expression levels of TE-expressed genes that were examined in the TEAD4 KD blastocysts; CDX2, GATA2 and GATA3, in the CCN2 KD blastocysts. Besides, the TEAD4 expression level was analyzed. Among these, the expression levels of CDX2, GATA2 and TEAD4, in CCN2 KD blastocysts significantly decreased compared to empty vector-injected controls, corresponding to TEAD4 KD blastocysts $(P<0.05)$ (Fig. 4C). Additionally, the fluorescent signals representing the TEAD4 proteins were reduced in the CCN2 KD blastocysts (Fig. 4D).

\section{Modulation of the ratio of TE to ICM cell numbers by CCN2 in bovine blastocysts}

CCN2 could control cell and tissue growth in various circumstances (Nakanishi et al. 2000, Ivkovic et al. 2003, Dornhöfer et al. 2006). Further, the TEAD4$\mathrm{CCN} 2$ regulation was involved in cell proliferation in human and mouse cell lines (Zhao et al. 2008). Considering these studies and our results, we performed immunofluorescent staining of CDX2 for the CCN2 KD

Table 1 Effect of TEAD4 KD on bovine preimplantation development in vitro.

\begin{tabular}{lccc}
\hline & & \multicolumn{2}{c}{ No of embryos developed to $(\% \pm$ S.E.M.) } \\
\cline { 3 - 4 } Treatment & Number of putative zygotes tested & 2-Cell & Blastocyst \\
\cline { 4 - 4 } Uninjected & 105 & $75(71.4 \pm 9.79)$ & $32(30.5 \pm 10.84)$ \\
Empty & 111 & $66(59.5 \pm 4.48)$ & $22(19.8 \pm 1.76)$ \\
shTEAD4 & 172 & $102(59.3 \pm 2.33)$ & $40(23.3 \pm 2.15)$ \\
\hline
\end{tabular}

Empty, embryos that vectors lacking shRNA sequence were injected into; S.E.M., standard error of the mean; shTEAD4, embryos that vectors containing TEAD4 shRNA sequence were injected into; uninjected, IVF embryos. 
A

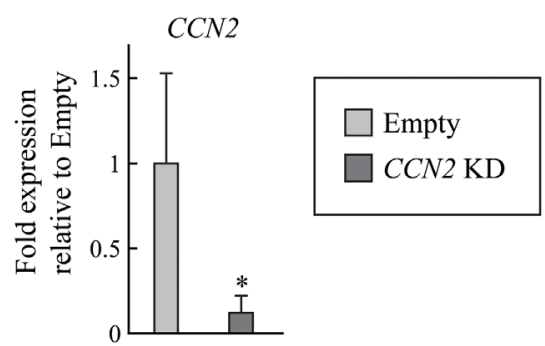

C

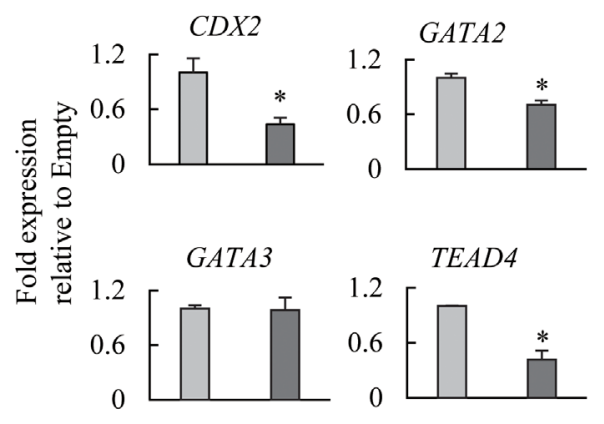

blastocysts to determine the effects of CCN2 KD on the cell numbers of CDX2-positive TE and CDX2-negative ICM. Although there were no significant differences in the TE, ICM and total cell numbers between controls and the CCN2 KD blastocysts (Fig. 5A), the ratio of TE to ICM cell numbers significantly decreased in the CCN2 KD blastocysts $(P<0.05)$ (Fig. 5B).

\section{Discussion}

It has been unclear whether the transcriptional modulation between TEAD4 and CCN2 is conserved in mammalian preimplantation embryos including cattle. We here attempted to dissect this by analyzing each TEAD4 and CCN2 KD bovine embryos. As expected, the TEAD4 KD induced the CCN2 suppression both at the mRNA and protein expression levels with decrease of the other genes required for TE cell characterization - CDX2 and GATA2. The decrease of CDX2 and GATA2

Table 2 Effect of CCN2 KD on bovine preimplantation development in vitro.

\begin{tabular}{llll}
\hline & $\begin{array}{l}\text { Number of } \\
\text { putative }\end{array}$ & & No of embryos developed to $(\% \pm$ S.E.M.) \\
\cline { 3 - 4 } Treatment & $\begin{array}{l}\text { zygotes } \\
\text { tested }\end{array}$ & 2 -cell & Blastocyst \\
\hline Uninjected & 171 & $129(75.4 \pm 9.10)$ & $45(26.3 \pm 7.43)$ \\
Empty & 182 & $143(78.6 \pm 5.79)$ & $41(22.5 \pm 2.08)$ \\
shCCN2 & 331 & $251(75.8 \pm 7.44)$ & $83(25.1 \pm 5.11)$ \\
\hline
\end{tabular}

Empty, embryos that vectors lacking shRNA sequence were injected into; S.E.M., standard error of the mean; shCCN2, embryos that vectors containing CCN2 shRNA sequence were injected into; uninjected, IVF embryos.

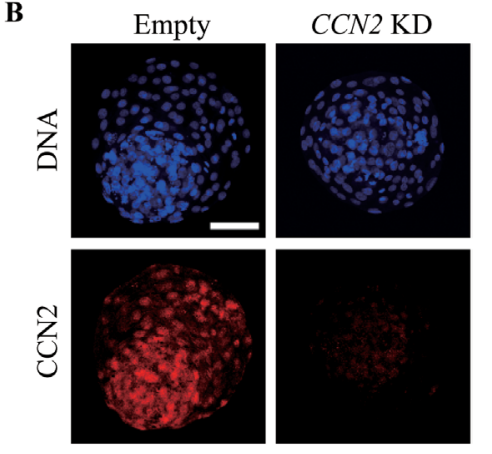

Figure 4 Effects of CCN2 knockdown (KD) on TE-expressed gene expression in bovine blastocysts. CCN2 KD efficiency was confirmed in blastocysts both at the mRNA (A) and protein (B) expression levels $(12.2 \%$ compared to Empty). (C) The relative expression levels of four TE-expressed genes in empty vector-injected control and CCN2 KD blastocysts (CDX2: 43.6\%, GATA2: 70.2\%, GATA3: $98.6 \%$, and TEAD4: $41.7 \%$ compared to Empty, respectively). (D) Immunofluorescent analysis of TEAD4 protein expression in the CCN2 KD blastocyst. Ten embryos were analyzed for each experiment. The values represent relative expression levels to empty vector-injected controls. The results are shown as means \pm S.E.M. (error bars) of three replicate experiments, $* P<0.05$. Scale bar $=100 \mu \mathrm{m}$.

expression was similarly observed in the CCN2 KD blastocysts, indicating that the modulation of CDX2 and GATA2 expression by TEAD4 was mediated through CCN2. Interestingly, the TEAD4 expression was also suppressed in the CCN2 KD blastocysts, suggesting that the TEAD4 expression was sustained by CCN2. Our results demonstrate that TEAD4 and CCN2 modulate mutual expression during mammalian blastocyst development, which was essential for the stable expression of the TE-expressed genes including CDX2

$\mathbf{A}$

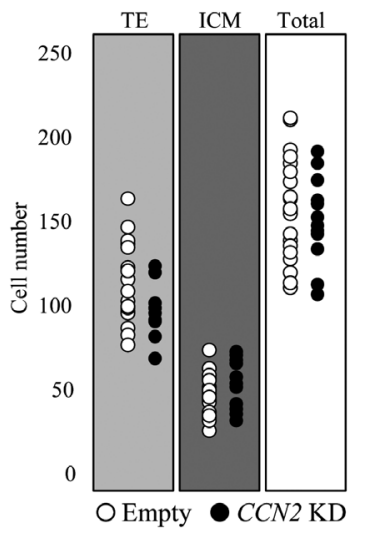

B

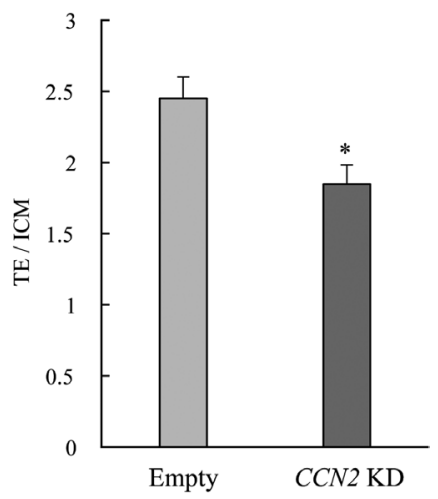

Figure $5 \mathrm{ICM} / \mathrm{TE}$ cell composition in the CCN2 KD blastocyst. (A) The numbers of TE (left), ICM (middle) and total (right) cells in the CCN2 KD blastocyst are shown. Each plot represents individual embryos; empty vector-injected controls (white) and CCN2 KD blastocysts (black), respectively. (B) Ratio of TE cells to ICM cells in empty vector-injected controls and CCN2 KD blastocysts. Error bar: S.E.M. $* P<0.05$. 
and GATA2. For a better understanding of mammalian blastocyst development, it would be interesting to discuss comparing these findings in bovine embryos with advanced studies resulted from various genetic approaches in mouse embryos.

In the bovine blastocyst, the TEAD4 mRNA expression was stronger in TE, and TEAD4 protein signals were also more distinct within nuclei in TE cells than in ICM. Characteristically, TEAD4 activates transcription of the target genes in cooperation with a transcriptional coactivator, Yes-associated protein (YAP) (Zhao et al. 2008). In mouse preimplantation embryos, YAP proteins localize into nuclei in outside blastomeres constructing TE, and thereby downstream factors of TEAD4 are specifically enhanced in TE (Nishioka et al. 2009). In bovine trophoblast CT-1 cells, YAP and TEAD4 coordinately regulate downstream factors (Kusama et al. 2016). As the CDX2 expression was downregulated in YAP KD blastocysts (Negrón-Pérez \& Hansen 2017), the regulatory mechanism for TE characterization through the YAP-TEAD4 axis may be conserved in bovine blastocyst as well as in mouse blastocysts. At least, the downregulation of CDX2, GATA2 and CCN2 in the TEAD4 KD blastocyst support that TEAD4 is associated with the expression of TE-expressed genes in the bovine blastocyst.

GATA3 is also one of the potent downstream targets of TEAD4 in mouse embryos (Ralston et al. 2010). However, in the present study, the GATA3 expression level was unchanged both in the TEAD4 and CCN2 $\mathrm{KD}$ embryos. These observations suggest the presence of upstream regulator (s) of GATA3 expression other than TEAD4 in the bovine blastocyst. This TEAD4independent regulation for GATA3 expression has been adopted in mouse embryos. For example, transcription factor AP-2, gamma (TCFAP2C) and EOMES had been shown to directly bind to the Gata3 promoter in mouse TS cells (Kidder \& Palmer 2010). Another group also reported that TEAD4 KD had no effects on GATA3 expression in cattle (Sakurai et al. 2017). Overall, TEAD4 might not be essential for the GATA3 mediation in the bovine blastocyst.

In several types of somatic cells, TEAD4 directly regulates the transcriptional activation of CCN2, resulting in cellular property alterations (Zhao et al. 2008, Lai et al. 2011). We also found that the CCN2 downregulation was caused by the TEAD4 suppression in bovine blastocysts. Furthermore, the TE-expressed genes downregulated and non-downregulated in the TEAD4 $\mathrm{KD}$ and $\mathrm{CCN} 2 \mathrm{KD}$ blastocysts were identical, suggesting that the sets of TE-expressed genes mediated by CCN2 and TEAD4 were overlapped. Nuclear localization of CCN2 could affect RNA transcription status in some cell types (Wahab et al. 2001). We also observed the CCN2 proteins within nuclei of bovine blastocysts as well as primary tissues in mice (Gray et al. 2007), and the protein expression was suppressed only in TE but not in ICM in the TEAD4 KD blastocysts. This might suggest that the CCN2 regulation through TEAD4 predominantly occurs in TE. Overall, this possible transcription regulation ability of CCN2 would play important roles for TE cell development in bovine blastocyst.

The significance of $\mathrm{CCN} 2$ has remained unclear in the blastocyst development. The analysis of TE, ICM and total cell numbers in the CCN2 KD blastocysts revealed that the CCN2 suppression significantly reduced the ratio of TE to ICM. We previously showed that the CCN2 expression level was greater in TE than that in ICM of bovine blastocysts (Nagatomo et al. 2013) as well as TEAD4 mRNA observed in the present study. This active CCN2 expression in TE might affect the regulation of the cell composition in bovine blastocysts. Recently, another group reported that the supplementation of human recombinant CCN2 into the culture medium for bovine embryos increased the ICM cell number (Kannampuzha-Francis et al. 2017). This apparent contradiction in effect on cell composition could be explained by the difference of CCN2 derivation. In the present study, we inhibited the endogenous CCN2 transcripts to investigate the autocrine effects of embryonic CCN2 expression on preimplantation development, which was inherently different from the addition of exogenous CCN2 proteins into the culture medium. As CCN2 expression was confirmed in bovine endometrial epithelial cells (Munoz et al. 2017), the supplementation of CCN2 recombinants might reflect the effects of maternal CCN2 on preimplantation development. Further analyses would be required for a deeper understanding of bovine CCN2 from maternal and embryonic sources in a dual manner.

In conclusion, our results demonstrate that the stable expression patterns of TE-expressed genes are maintained through reciprocal regulation of both TEAD4 and CCN2 in cattle. To our knowledge, this is the first study showing CCN2 expression mediated by TEAD4 in mammalian embryos. Further analyses of interaction between TEAD4 and CCN2 in bovine blastocysts would expand the understanding of phenomena underling proper cell differentiation in preimplantation development.

\section{Supplementary data}

This is linked to the online version of the paper at https://doi.org/10.1530/REP-18-0043.

\section{Declaration of interest}

The authors declare that there is no conflict of interest that could be perceived as prejudicing the impartiality of the research reported. 


\section{Funding}

This work was supported in part by a Grant-in-Aid for Challenging Exploratory Research to M K (No. 16K14587) from the Ministry of Education, Culture, Sports, Science and Technology of Japan.

\section{References}

Akizawa H, Nagatomo H, Odagiri H, Kohri N, Yamauchi N, Yanagawa Y, Nagano M, Takahashi M \& Kawahara M 2016 Conserved roles of fibroblast growth factor receptor 2 signaling in the regulation of inner cell mass development in bovine blastocysts. Molecular Reproduction and Development 83 516-525. (https://doi.org/10.1002/mrd.22646)

Andey T, Marepally S, Patel A, Jackson T, Sarkar S, Connell MO, Reddy RC, Chellappan S, Singh P \& Singh M 2014 Cationic lipid guided shorthairpin RNA interference of annexin A2 attenuates tumor growth and metastasis in a mouse lung cancer stem cell model. Journal of Controlled Release 184 67-78. (https://doi.org/10.1016/j.jconrel.2014.03.049)

Aono A, Nagatomo H, Takuma T, Nonaka R, Ono Y \& Kawahara M 2013 Dynamics of intracellular phospholipid membrane organization during oocyte maturation and successful vitrification of immature oocytes retrieved by ovum pick-up in cattle. Theriogenology 79 1146.e1-1152. e1. (https://doi.org/10.1016/j.theriogenology.2013.02.009)

Arnold SJ \& Robertson EJ 2009 Making a commitment: cell lineage allocation and axis patterning in the early mouse embryo. Nature Reviews Molecular Cell Biology 10 91-103. (https://doi.org/10.1038/ nrm2618)

Berg DK, Smith CS, Pearton DJ, Wells DN, Broadhurst R, Donnison M \& Pfeffer PL 2011 Trophectoderm lineage determination in cattle. Developmental Cell 20 244-255. (https://doi.org/10.1016/j. devcel.2011.01.003)

Bou G, Liu S, Sun M, Zhu J, Xue B, Guo J, Zhao Y, Qu B, Weng X, Wei Y et al. 2017 CDX2 is essential for cell proliferation and polarity in porcine blastocysts. Development 144 1296-1306. (https://doi.org/10.1242/ dev.141085)

Brackett BG \& Oliphant G 1975 Capacitation of rabbit spermatozoa in vitro. Biology of Reproduction 274 260-274. (https://doi.org/10.1095/ biolreprod 12.2.260)

Chawiengsaksophak K, James R, Hammond VE, Köntgen F \& Beck F 1997 Homeosis and intestinal tumours in $\mathrm{Cd} \times 2$ mutant mice. Nature 386 84-87. (https://doi.org/10.1038/386084a0)

Dornhöfer N, Spong S, Bennewith K, Salim A, Klaus S, Kambham N, Wong C, Kaper F, Sutphin P, Nacalumi R et al. 2006 Connective tissue growth factor-specific monoclonal antibody therapy inhibits pancreatic tumor growth and metastasis. Cancer Research 66 5816-5827. (https:// doi.org/10.1158/0008-5472.CAN-06-0081)

Gray MR, Malmquist JA, Sullivan M, Blea M \& Castellot JJ Jr 2007 CCN5 Expression in mammals. II. Adult rodent tissues. Journal of Cell Communication and Signaling 1 145-158. (https://doi.org/10.1007/ s12079-007-0013-z)

Gulnaar AS, Amy KW \& David RB 1998 Localization of connective tissue growth factor during the period of embryo implantation in the mouse. Biology of Reproduction 59 1207-1213. (https://doi.org/10.1095/ biolreprod59.5.1207)

Home P, Saha B, Ray S, Dutta D, Gunewardena S, Yoo B, Pal A, Vivian JL, Larson M, Petroff M et al. 2012 Altered subcellular localization of transcription factor TEAD4 regulates first mammalian cell lineage commitment. PNAS 109 7362-7367. (https://doi.org/10.1073/ pnas.1201595109)

Home P, Kumar RP, Ganguly A, Saha B, Milano-Foster J, Bhattacharya B, Ray S, Gunewardena S, Paul A, Camper SA et al. 2017 Genetic redundancy of GATA factors in extraembryonic trophoblast lineage ensures progression of both pre and postimplantation mammalian development. Development 144 876-888. (https://doi.org/10.1242/ dev.145318)

Ivkovic S, Yoon BS, Popoff SN, Safadi FF, Libuda DE, Stephenson RC, Daluiski A \& Lyons KM 2003 Connective tissue growth factor coordinates chondrogenesis and angiogenesis during skeletal development. Development 130 2779-2791. (https://doi.org/10.1242/dev.00505)
Kannampuzha-Francis J, Tribulo P \& Hansen PJ 2017 Actions of activin A, connective tissue growth factor, hepatocyte growth factor and teratocarcinoma-derived growth factor 1 on the development of the bovine preimplantation embryo. Reproduction, Fertility and Development 29 1329-1339. (https://doi.org/10.1071/RD16033)

Kidder BL \& Palmer S 2010 Examination of transcriptional networks reveals an important role for TCFAP2C, SMARCA4, and EOMES in trophoblast stem cell maintenance. Genome Research 20 458-472. (https://doi. org/10.1101/gr.101469.109)

Kuijk EW, van Tol LTA, Van de Velde H, Wubbolts R, Welling M, Geijsen N \& Roelen BAJ 2012 The roles of FGF and MAP kinase signaling in the segregation of the epiblast and hypoblast cell lineages in bovine and human embryos. Development 139 871-882. (https://doi.org/10.1242/ dev.071688)

Kusama K, Bai R, Sakurai T, Bai H, Ideta A, Aoyagi Y \& Imakawa K 2016 A transcriptional cofactor YAP regulates IFNT expression via transcription factor TEAD in bovine conceptuses. Domestic Animal Endocrinology $\mathbf{5 7}$ 21-30. (https://doi.org/10.1016/j.domaniend.2016.05.002)

Lai D, Ho KC, Hao Y \& Yang X 2011 Taxol resistance in breast cancer cells is mediated by the hippo pathway component TAZ and its downstream transcriptional targets Cyr61 and CTGF. Cancer Research 71 2728-2738. (https://doi.org/10.1158/0008-5472.CAN-10-2711)

Li W, Ou Q, Dai H \& Liu C 2015 Lentiviral-mediated short hairpin RNA knockdown of MTDH inhibits cell growth and induces apoptosis by regulating the PTEN/AKT pathway in hepatocellular carcinoma. International Journal of Molecular Science 16 19419-19432. (https://doi. org/10.3390/ijms160819419)

Mitsui K, Tokuzawa Y, Itoh H, Segawa K, Murakami M, Takahashi K, Maruyama M, Maeda M \& Yamanaka S 2003 The homeoprotein Nanog is required for maintenance of pluripotency in mouse epiblast and ES cells. Cell 113 631-642. (https://doi.org/10.1016/S0092-8674(03)00393-3)

Munoz M, Martin D, Carrocera S, Alonso-Guervos M, Mora MI, Corrales FJ, Peynot N, Giraud-Delville C, Duranthon V, Sandra O et al. 2017 Localisation of stem cell factor, stanniocalcin-1, connective tissue growth factor and heparin-binding epidermal growth factor in the bovine uterus at the time of blastocyst formation. Reproduction, Fertility and Development 29 2127-2139.

Nagatomo H, Kagawa S, Kishi Y, Takuma T, Sada A, Abe Y, Wada Y, Takahashi M \& Kono T 2013 Transcriptional wiring for establishing cell lineage specification at the blastocyst stage in cattle. Biology of Reproduction 88. (https://doi.org/10.1095/biolreprod.113.108993)

Nagatomo H, Akizawa H, Sada A, Kishi Y, Yamanaka KI, Takuma T, Sasaki K, Yamauchi N, Yanagawa Y, Nagano M et al. 2015 Comparing spatial expression dynamics of bovine blastocyst under three different procedures: in-vivo, in-vitro derived, and somatic cell nuclear transfer embryos. Japanese Journal of Veterinary Research 63 159-171.

Nagatomo H, Kohri N, Akizawa H, Hoshino Y, Yamauchi N, Kono T, Takahashi M \& Kawahara M 2016 Requirement for nuclear autoantigenic sperm protein mRNA expression in bovine preimplantation development. Animal Science Journal 87 457-461. (https://doi.org/10.1111/asj.12538)

Nakanishi T, Nishida T, Shimo T, Kobayashi K, Kubo T, Tamatani T, Tezuka K \& Takigawa M 2000 Effects of CTGF/Hcs24, a product of a hypertrophic chondrocyte-specific gene, on the proliferation and differentiation of chondrocytes in culture. Endocrinology 141 264-273. (https://doi. org/10.1210/endo.141.1.7267)

Negrón-Pérez VM \& Hansen PJ 2017 Role of Yes-associated protein 1, angiomotin and mitogen activated kinase kinase $1 / 2$ in development of the bovine blastocyst. Biology of Reproduction 2 170-183. (https://doi. org/10.1093/biolre/iox172)

Nelson NG, Skeie JM, Muradov H, Rowell HA \& Seo S 2014 CAPN5 gene silencing by short hairpin RNA interference. BMC Research Notes 7 1-7. (https://doi.org/10.1186/1756-0500-7-1)

Niakan KK \& Eggan K 2013 Analysis of human embryos from zygote to blastocyst reveals distinct gene expression patterns relative to the mouse. Developmental Biology 375 54-64. (https://doi.org/10.1016/j. ydbio.2012.12.008)

Nichols J, Zevnik B, Anastassiadis K, Niwa H, Klewe-Nebenius D, Chambers I, Scholer H \& Smith A 1998 Formation of pluripotent stem cells in the mammalian embryo dependes on the POU transcription factor Oct4. Cell 95 379-391. (https://doi.org/10.1016/S00928674(00)81769-9) 
Nishioka N, Yamamoto S, Kiyonari H, Sato H, Sawada A, Ota M, Nakao K \& Sasaki H 2008 Tead4 is required for specification of trophectoderm in pre-implantation mouse embryos. Mechanisms of Development 125 270-283. (https://doi.org/10.1016/j.mod.2007.11.002)

Nishioka N, Inoue K, Adachi K, Kiyonari H, Ota M, Ralston A, Yabuta N, Hirahara S, Stephenson RO, Ogonuki N et al. 2009 The hippo signaling pathway components Lats and Yap pattern Tead4 activity to distinguish mouse trophectoderm from inner cell mass. Developmental Cell $\mathbf{1 6}$ 398-410. (https://doi.org/10.1016/j.devcel.2009.02.003)

Niwa H, Toyooka Y, Shimosato D, Strumpf D, Takahashi K, Yagi R \& Rossant J 2005 Interaction between Oct3/4 and Cdx2 determines trophectoderm differentiation. Cell $\mathbf{1 2 3}$ 917-929. (https://doi. org/10.1016/j.cell.2005.08.040)

Orimo A, Gupta PB, Sgroi DC, Arenzana-Seisdedos F, Delaunay T, Naeem R, Carey VJ, Richardson AL \& Weinberg RA 2005 Stromal fibroblasts present in invasive human breast carcinomas promote tumor growth and angiogenesis through elevated SDF-1/CXCL12 secretion. Cell 121 335-348. (https://doi.org/10.1016/j.cell.2005.02.034)

Ralston A, Cox BJ, Nishioka N, Sasaki H, Chea E, Rugg-Gunn P, Guo G, Robson P, Draper JS \& Rossant J 2010 Gata3 regulates trophoblast development downstream of Tead 4 and in parallel to Cdx2. Development 137 395-403. (https://doi.org/10.1242/dev.038828)

Sakurai N, Takahashi K, Emura N, Hashizume T \& Sawai K 2017 Effects of downregulating TEAD4 transcripts by RNA interference on early development of bovine embryos. Journal of Reproduction and Development 63 135-142. (https://doi.org/10.1262/jrd.2016-130)
Strumpf D, Mao CA, Yamanaka Y, Ralston A, Chawengsaksophak K, Beck F \& Rossant J $2005 \mathrm{Cdx} 2$ is required for correct cell fate specification and differentiation of trophectoderm in the mouse blastocyst. Development 132 2093-2102. (https://doi.org/10.1242/dev.01801)

Wahab NA, Brinkman H \& Mason RM 2001 Uptake and intracellular transport of the connective tissue growth factor: a potential mode of action. Biochemical Journal 359 89-97. (https://doi.org/10.1042/ bj3590089)

Yagi R, Kohn MJ, Karavanova I, Kaneko KJ, Vullhorst D, DePamphilis ML \& Buonanno A 2007 Transcription factor TEAD4 specifies the trophectoderm lineage at the beginning of mammalian development. Development 134 3827-3836. (https://doi.org/10.1242/dev.010223)

Zhao B, Ye X, Yu J, Li L, Li W, Li S, Yu J, Lin JD, Wang C, Chinnaiyan AM et al. 2008 TEAD mediates YAP-dependent gene induction and growth control. Genes and Development 22 1962-1971. (https://doi. org/10.1101/gad.1664408)

Received 21 January 2018

First decision 15 February 2018

Revised manuscript received 5 April 2018

Accepted 16 April 2018 